\title{
Astrodynamics Science about Itokawa, Gravity and Ephemeris
}

\author{
M. Yoshikawa , H. Ikeda, H. Yano, J. Saito, T. Kubota, T. Hashimoto, A. Fujiwara, J. Kawaguchi \\ Japan Aerospace Exploration Agency, Sagamihara, Kanagawa, 229-8510, Japan \\ T. Kominato, M. Matsuoka, K. Shirakawa \\ NEC Aerospace Systems Ltd, Yokohama, Kanagawa, 224-0053, Japan \\ T. Ohnishi \\ Fujitsu Ltd, Chiba, Chiba, 261-8588, Japan \\ S. Abe, T. Mukai \\ Kobe University, Nada, Kobe, 657-8501, Japan \\ R. Gaskell \\ Jet Propulsion Laboratory, Pasadena, California, 91109 \\ and \\ D. Scheeres \\ The University of Michigan, Ann Arbor, Michigan, 48109-2140
}

\begin{abstract}
[Abstract] Hayabusa was moving near Asteroid Itokawa for about three months in 2005. Using tracking and navigation data such as range, Doppler, distance from the asteroid, and optical images taken by navigation cameras, we estimated the mass of Itokawa for several orbital phases. The results are consistent in their error ranges. The estimated mass of Itokawa is $3.51 \pm 0.105\left(10^{10} \mathrm{~kg}\right)$, from which the density of Itokawa is derived as $1.9 \pm 0.13$ $\left(\mathrm{g} / \mathrm{cm}^{3}\right)$. This is one of the important characteristics to understand the nature of Itokawa. We are also trying to investigate the accuracy of the ephemeris of this asteroid.
\end{abstract}

\section{Introduction}

$\mathrm{T}$ HE Hayabusa mission is the asteroid sample return mission of Japan. The spacecraft was launched on May 9, 2003, and after the Earth swingby on May 19, 2004, it arrived at its target asteroid, (25143) Itokawa, on September 12, 2005. Before the arrival, it was already known by ground-based observation that the size of Itokawa is rather small, about $500 \mathrm{~m}$ in length. This is correct. What Hayabusa saw was certainly a very tiny object, but the appearance of its surface was completely unexpected. On its surface, there are few craters but a lot of boulders. Figure 1 shows the image of Hayabusa mission and the photograph of asteroid Itokawa. The first scientific results by Hayabusa were summarized in a special issue of Science Journal ${ }^{1}$.

The arrival point of Hayabusa on September 12, 2005 was about $20 \mathrm{~km}$ away from the surface of Itokawa. We call this position as "Gate position." At first, Hayabusa was moving around this gate position, and gradually it went down toward the asteroid. At the end of September 2005, Hayabusa arrived at the position of about $7 \mathrm{~km}$ from the asteroid. We call this position as "Home position." Hayabusa did not revolve around the asteroid, but it moved along the line that connects the earth and the asteroid. In October 2005, Hayabusa was located around the Home position, and toward the end of October, Hayabusa approached less than $4 \mathrm{~km}$ from the surface of Itokawa. Until this time, we have almost finished the basic observations of Itokawa. We have had information about the surface of Itokawa and we have constructed the shape model of Itokawa. Also, we have selected the candidate places for touchdown. Thus, at the end of October, we were ready for touchdown.

\footnotetext{
*Associate Professor, Institute of Space and Astronautical Science.
} 

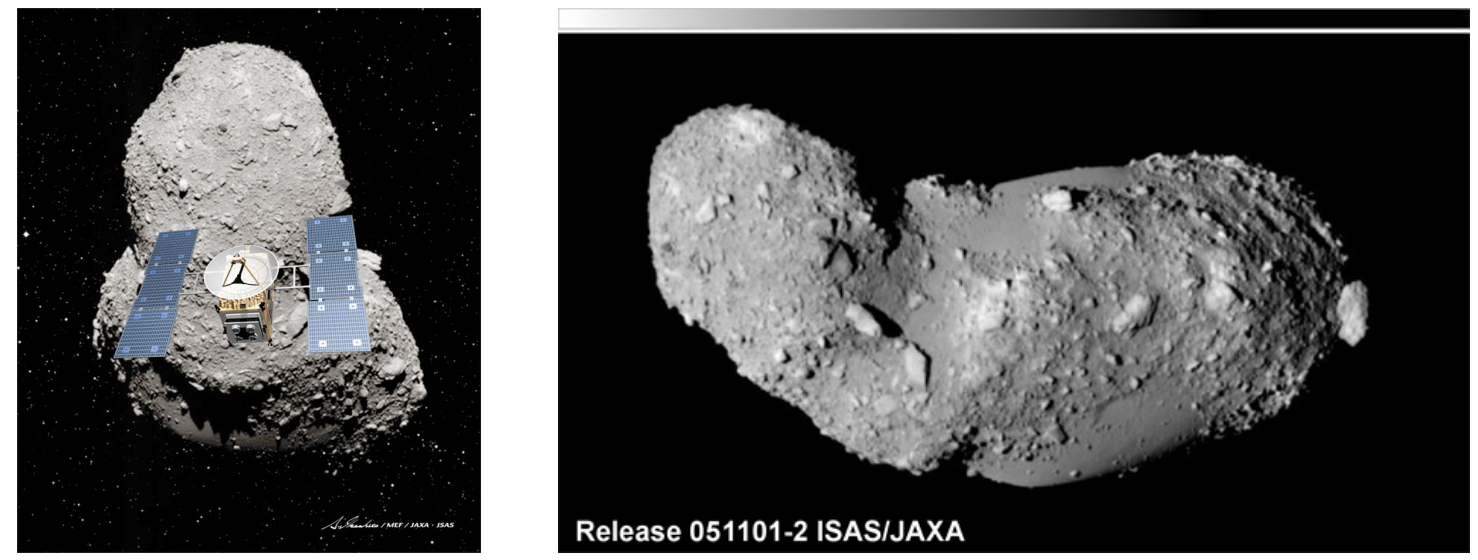

Figure 1. Hayabusa mission and Asteroid Itokawa. Left is the composite image of Itokawa (photograph) and Hayabusa (illustration). Right is one of the photographs, which shows strange nature of Itokawa. The illustration was made by A. Ikeshita.

In November 2005, Hayabusa tried touching down. The first touchdown rehearsal was executed on November 4, 2005. In this first descent to Itokawa, we encountered several problems that we did not expect, so we carried out two more descent operations on November 9 and 12. Then we had confidence to carry out touchdown, and the first touchdown was executed on November 20. In this first touch down, also totally unexpected things happened. We learned later that Hayabusa stayed on the surface of Itokawa more than 30 minutes. However, the sapling sequence was not performed in this first touchdown, so we tried second touchdown on November 26. At this second touchdown, the mission sequence was perfect, so all of us thought that Hayabusa was successful in getting the surface material. However, after the liftoff from Itokawa, the fuel leak occurred. We were forced to operate Hayabusa in very difficult status and we were not able to confirm that the bullet was fired at the moment of the second touchdown. Anyway, at present (July 2006) we are able to operate Hayabusa, and we think we can make it return to the earth in June 2010, which is three years behind the schedule. We hope some of the surface materials are inside the capsule.

This is the short history of Hayabusa. We have learned a lot of engineering matters as well as we have discovered many scientifically new things. One of them is the mass of the asteroid. The mass of the asteroid is important not only for the science of Itokawa but also for the navigation of the spacecraft around Itokawa. In fact, when Hayabusa was near Itokawa, the Hayabusa navigation team estimated the mass of Itokawa, because they need it for the daily operation. However the estimated mass in such daily operation was not accurate enough, so in this paper, we do not mention about this real time mass estimation. But here we summarize the mass estimations, which were done after the mission phase, in chapter II. We also show some data, which may be important when we analyze the orbit of Itokawa, in chapter III.

\section{Mass Estimation of Itokawa}

Several teams tried to estimate the mass of Itokawa by using different data and different methods. In this section, we summarize the results of these mass estimations. Figure 2 shows the schematic image of the mass estimation. Basically Hayabusa was moving along the line that connects the earth and Itokawa. When Hayabusa was near Itokawa, the sun and Earth located almost same direction from Hayabusa. The forces that affect on Hayabusa are the gravity from Itokawa, the solar radiation pressure, and the gravity from other celestial bodies, such as the sun and planets. The gravitational accelerations from the Sun and the planets are well known, so we should estimate the solar radiation pressure and the gravitational attraction from Itokawa separately. Then we can know the mass of Itokawa. The data used for mass estimation is the range and Doppler from ground stations, the distance between Hayabusa and the surface of Itokawa measured by LIDAR (Light Detection and Ranging Instrument), and the angle of Itokawa form Hayabusa obtained by the navigation cameras.

Figure 3 shows that the variation of the distance between Hayabusa and Itokawa. The motion of Hayabusa, which was mentioned in the previous section, can be understood clearly in this figure. In Fig.3, the periods when the mass estimations were carried out are marked by circles of A to E. Here we briefly summarize what were done in each phase. (Please see the papers of each team for the analysis and the result in detail.) 


\section{A. Mass Estimation around Gate Position and Home Position}

The first mass estimation was done when Hayabusa moved around the gate position and the home position. This period is shown by "A" in Fig.3. Hayabusa had acceleration toward the asteroids, partly due to the gravitational attraction from Itokawa, but mainly due to the solar radiation pressure. When Hayabusa is around the gate position (about $20 \mathrm{~km}$ from Itokawa), the effect of the solar radiation pressure is about 20 times larger than that of the gravitational attraction by Itokawa. The motion of Hayabusa in this period is something like free fall in one dimension in a homogeneous gravitational field. In order not to approach very close to the asteroid, sometimes upward thrusts were executed, which are shown as the changes of direction of motion in Fig.3.

Using the range and Doppler data, the mass of Itokawa was estimated. The estimation error is about $15 \%$. This error is rather large because in this period, the effect of the gravitational attraction by Itokawa is much smaller than that of the solar radiation pressure. In Fig.4, the actual orbit of Hayabusa of this period is shown. The detailed result of analysis was presented in a separate paper ${ }^{2}$.

\section{B. Mass Estimation at $3.5 \mathrm{~km}$ from Itokawa}

The mass estimation in the period "A" was successful, so we expected that we would perform much precise mass estimation when the spacecraft approached the asteroid much closer. However at the beginning of October 2005 (just after the period "A" in Fig.3), one reaction wheel had a trouble and stopped functioning. Hayabusa has three reaction wheels, and one of them broke down before arriving at the asteroid. The attitude control did not have serious problems with two reaction wheels. However, by the break down of the second reaction wheel, we must use the chemical thrusters to control the attitude of Hayabusa. When chemical thrusters are used, small acceleration is generated in its orbital motion. This makes very difficult to estimate the mass of Itokawa.

In order to get the proper orbital data to estimate the mass, we intentionally stop the attitude control for two days, and let the Hayabusa move without the artificial force. This period is October 21 and 22, which is shown by "B" in Fig.3. The orbit at this period is a part of the trajectory that revolves around the asteroid (Fig.4). Using the data of LIDAR and the navigation camera in addition to the range and Doppler, the mass of Itokawa was estimated with the error of $5 \%$. The detailed analysis is shown in separate paper ${ }^{3}$.

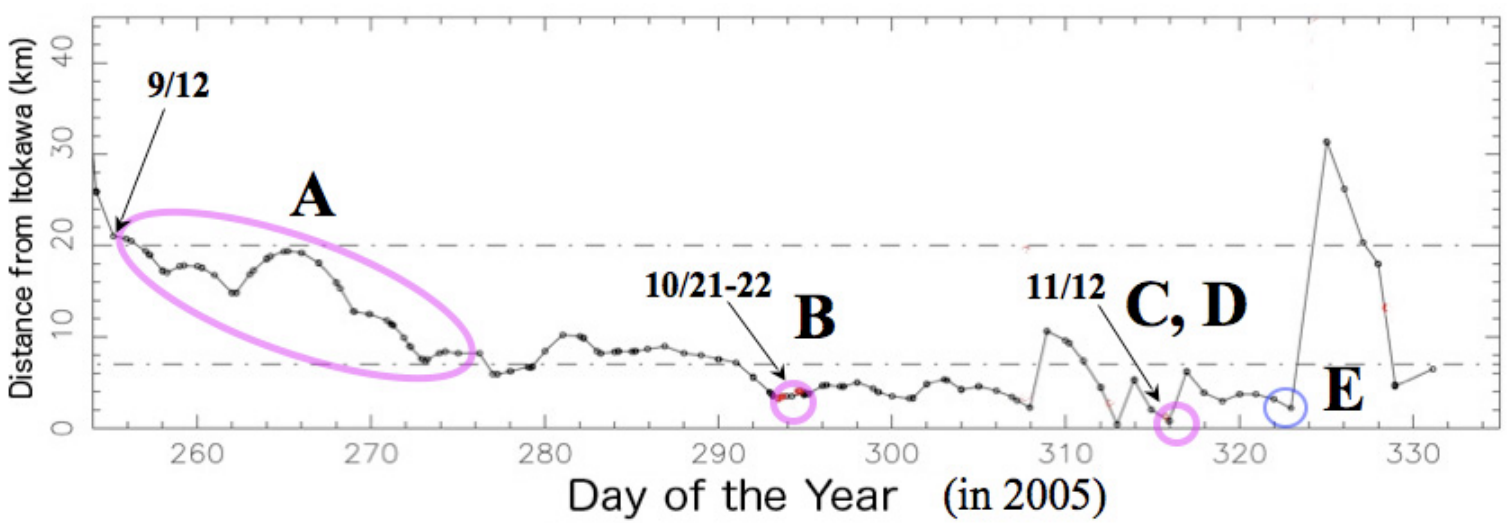

Figure 3. Variation of distance between Hayabusa and Itokawa and the periods when the mass estimations ware carried out. The circles marked by " $A$ " to " $E$ " indicate the periods when the mass of Itokawa was estimated. The data in November 2005 (after DOY of 307) does not represent correct change because the data points are not dense enough. The distance data were based on the ranging measurement. 


\section{Mass Estimation at Descent Rehearsal}

When Hayabusa locates quite near to Itokawa, the gravitational attraction by Itokawa is much more stronger than the solar radiation pressure. As we mentioned in Chapter I, Hayabusa made approaches to Itokawa five times in November 2005. By using the data (mainly LIDAR and navigation camera) of third descent (Nov. 12), two independent mass estimations were carried out. They are "C" and "D" in Fig.3. Each mass estimation was done by using the data of short period, and the corresponded orbits are shown in Fig.5. These two segments of the orbit are so close to the asteroid that the mass distribution (shape of the asteroid) was taken into account assuming that the density of asteroid is homogeneous. (In the analysis of "A" and "B", the asteroid is treated as a point mass.) The error of the mass estimation of " $\mathrm{C}$ " and " $\mathrm{D}$ " is 5 or $6 \%$. The detailed results are presented in Ref. 4 for the case "C", and Ref.5 for the case "D".

\section{Local Gravity at Surface of Itokawa}

At the first touchdown marked by "E" in Fig.3, Hayabusa was put in a status of free fall unexpectedly, and it bounced twice on the surface of Itokawa. Therefore, if we know the change of distance between the spacecraft and the surface of Itokawa, we can calculate the local gravitational acceleration. Actually we have data of distance by LRF (Laser Range Finder), but we must be careful to use this data because this data does not necessarily represent the vertical distance. We must calibrate the data by considering the attitude of the spacecraft, the orbit, and the shape of the asteroid. The analysis is still ongoing, so we do not mention the result of this analysis here. The behavior of Hayabusa at this touchdown is reported in Ref.6.

\section{E. Summary of Mass Estimation}

The mass estimations of Itokawa are summarized in Table 1, where the estimated values are shown in GM (G is the gravitational constant and $\mathrm{M}$ is the mass of Itokawa). The estimate errors are also shown in Table 1. In order to compare these results, the error range of each estimate is shown in Fig.6. From this figure, we can say that the estimated values are consistent in each other.

Taking the weighted mean of these results, the final value of $\mathrm{GM}$ is $2.34 \pm 0.07\left(10^{-9} \mathrm{~km}^{3} / \mathrm{s}^{2}\right)$, which is converted to the mass of Itokawa as $3.51 \pm 0.105\left(10^{10} \mathrm{~kg}\right)$. According to the shape modeling team ${ }^{7}$, the volume of Itokawa was estimated as $1.84 \pm 0.092\left(10^{7} \mathrm{~m}^{3}\right)$. Thus the density of Itokawa is calculated as $1.9 \pm 0.13\left(\mathrm{~g} / \mathrm{cm}^{3}\right)$. This value of density is quite important to understand the nature of Itokawa (This is briefly mentioned in the last chapter). This result of the mass and the density was taken as the first reference of the basic physical characteristics of Itokawa and they are reported in Ref.8.

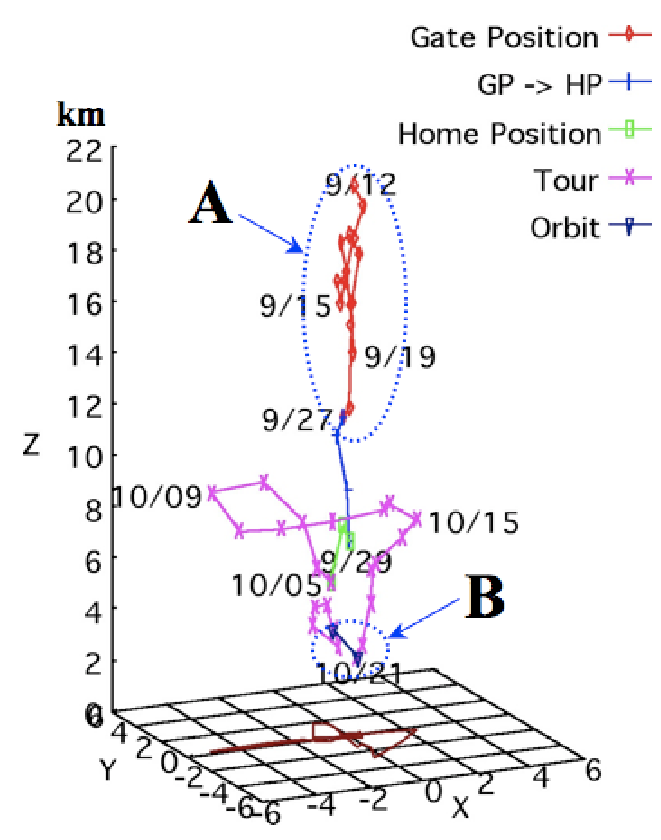

Figure 4. Orbit of Hayabusa from the asteroid arrival to the end of October. The earth locates in the positive direction of the z-axis. Itokawa is at the Origin. For some points, the dates are written as Month/Date.

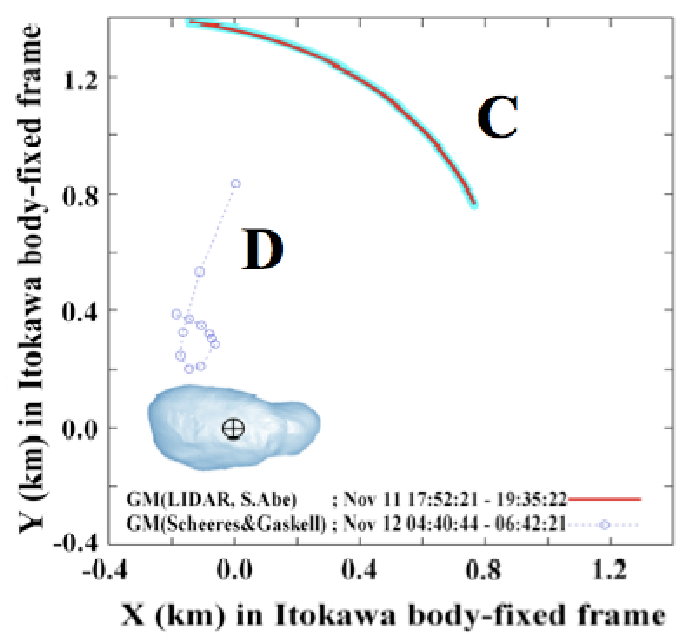

Figure 5. Orbits of Hayabusa, where the mass of Itokawa was estimated. These two segments are a part of the orbit of Hayabusa for the descent operation on November 12, 2005. Using the data of these passes, the mass of Itokawa was estimated individually. 
Table 1 Results of Mass Estimation of Asteroid Itokawa

\begin{tabular}{ccccccc}
\hline Phase & Period & Data Type* & $\begin{array}{c}\text { Distance from } \\
\text { Itokawa }\end{array}$ & $\begin{array}{c}\text { Model of } \\
\text { Itokawa }\end{array}$ & $\begin{array}{c}\text { GM } \\
10^{-9} \mathrm{~km}^{3} / \mathrm{s}^{2}\end{array}$ & Error \\
\hline A & Sep. 12- Oct. 2 & R, Dop. & $20-7 \mathrm{~km}$ & point mass & 2.34 & $15 \%$ \\
B & Oct. 21-22 & R, Dop., Opt, LI & $3.5 \mathrm{~km}$ & point mass & 2.29 & $5 \%$ \\
C & Nov. 12 & LI, Opt. & $1427-825 \mathrm{~m}$ & polyhedron & 2.39 & $5 \%$ \\
D & Nov. 12 & Opt., LI & $800-100 \mathrm{~m}$ & polyhedron & 2.36 & $6 \%$ \\
E & Nov. 19 & LRF & $20-10 \mathrm{~m}$ & - & - & - \\
\hline
\end{tabular}

*R: Range, Dop: Doppler, LI: LIDAR, Opt: Optical images, LRF: Laser Range Finder

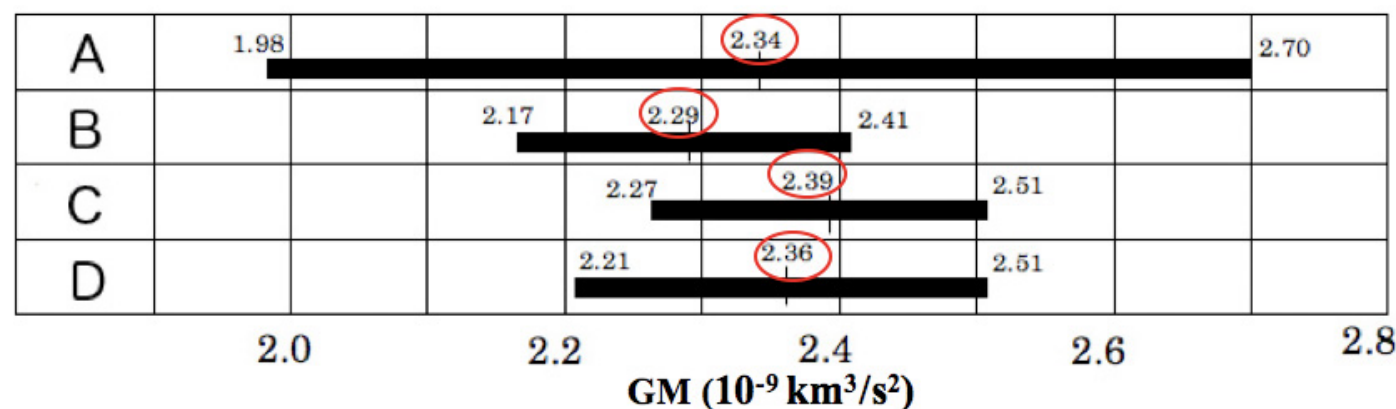

Figure 6. Estimated GM values with the error range. The values of GM and error in Table 1 are shown graphically. The dark lines indicate the range of estimated GM for each phase of the orbit.

\section{Orbital Information of Itokawa}

The mass of the asteroid is the most important thing in astrodynamics science of Hayabusa. Another interesting issue related astrodynamics is the orbit of Itokawa. The orbit of Itokawa was determined quite well before Hayabusa's arrival, because there were radar observations as well as a lot of ground-based optical observations. According to the information from Jet Propulsion Laboratory, the uncertainty in the ephemeris of Itokawa is of order $7 \mathrm{~km}(1 \sigma)$. This is quite good in accuracy but we tried to find difference between the observed position and the position calculated from the ephemeris of Itokawa.

One of the attempts is illustrated in Fig.7. We measure the range of Hayabusa from the earth $(\mathrm{O})$ and we know the range of Itokawa from the ephemeris (C). We have the distance data between Hayabusa and the surface of

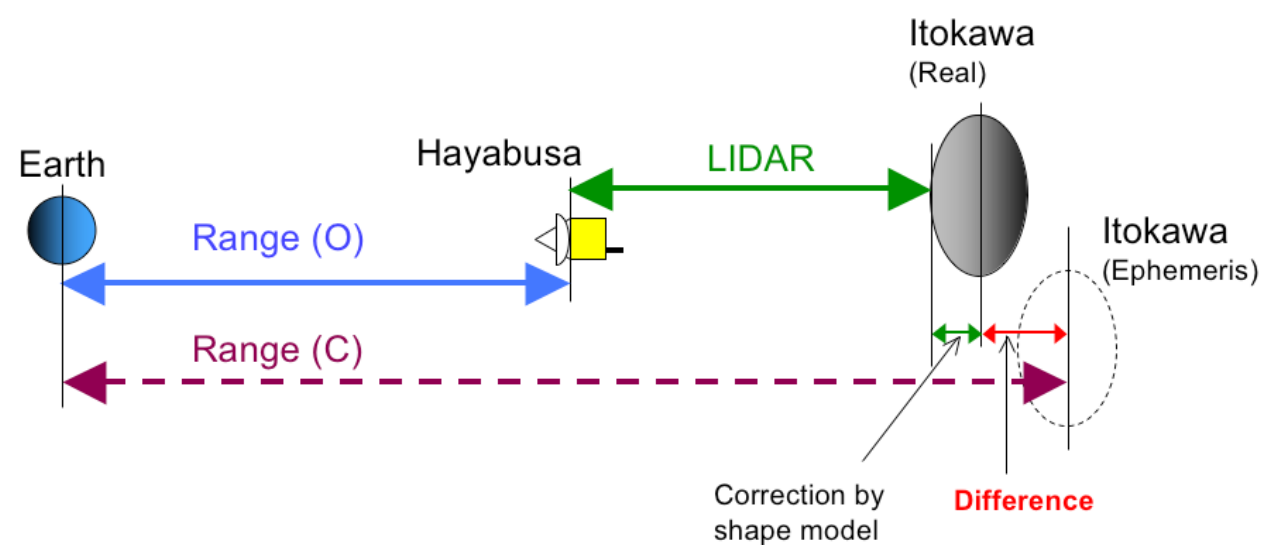

Figure 7. Schematic figure of distance measurements. We try to detect the difference between the position of the observed Itokawa and that of the ephemeris. 


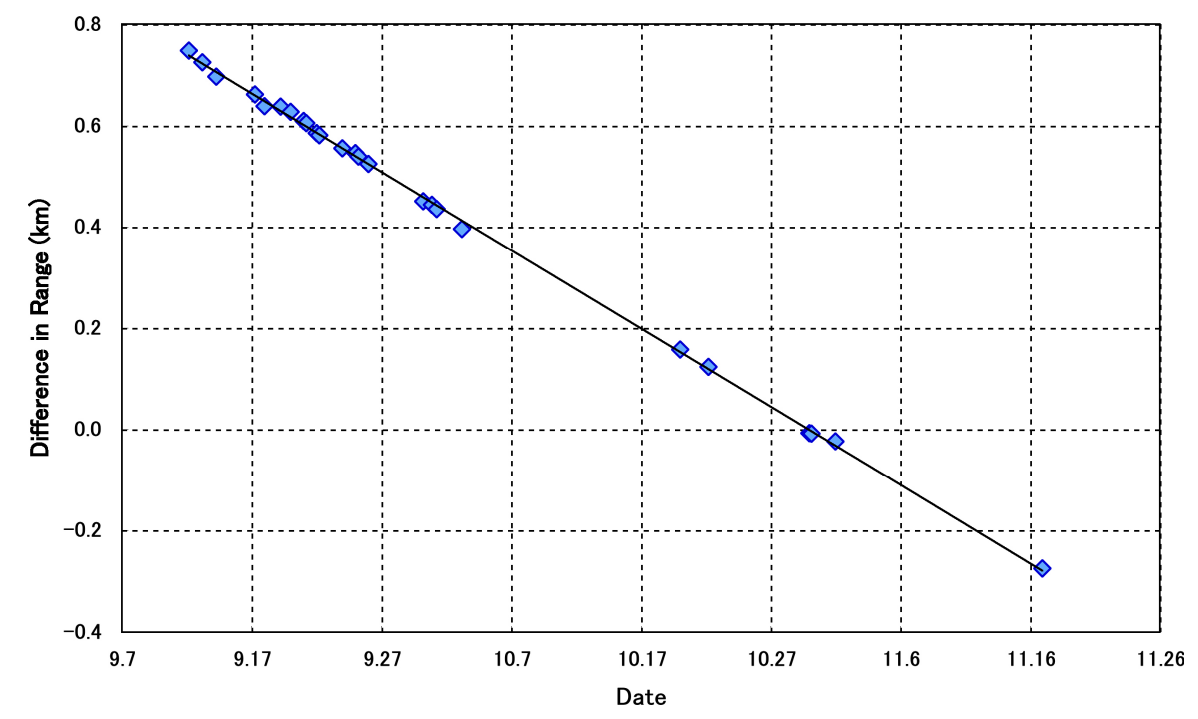

Figure 8. Difference in range between the observed Itokawa and the Itokawa in ephemeris. The plots are the detected difference and the line is the least square fit.

Itokawa by LIDAR, and we have the shape model of Itokawa. Therefore, we can calculate the difference in the line of sight between the observed Itokawa and the ephemeris of Itokawa. The result is shown in Fig.8. There is a systematic change in this difference, which should be explained either by the modification of the orbit of Itokawa or by some other reasons.

Another attempt that we made was the Delta-VLBI (Very Long Baseline Interferometry) observation at the descent and touchdown operations. At these operations, Hayabusa located just in front of Itokawa, so we can measure the Itokawa's direction from the earth by observing Hayabusa. The data is still under investigation, but the preliminary results show that the measured position is consistent with the position of ephemeris with in the error of 6 mas (milli-arc-seconds), which is about $10 \mathrm{~km}$ in vertical direction to the line of sight.

As for the orbit of Itokawa, we have not reached to final results. This is one of our future problems.

\section{Summary}

The mass of Itokawa was estimated by using tracking and navigation data of Hayabusa. Four teams tried to estimate the mass by using different data and different methods. The obtained results are consistent within the error ranges, and the mass of Itokawa was determined as $3.51 \pm 0.105\left(10^{10} \mathrm{~kg}\right)$. The estimated error is $3 \%$. This value of the mass is converted the density of Itokawa, and it is $1.9 \pm 0.13\left(\mathrm{~g} / \mathrm{cm}^{3}\right)$. This indicates that the macroporosity of Itokawa is about $40 \%^{8}$. This result is quite important when we study the origin, evolution, and structure of Itokawa. One of the possible scenarios is as follows: (1) The parent body was disrupted by an impact of another asteroid. (2) A portion of fragments coagulated each other forming two small bodies. (3) These two bodies were merged and Itokawa was born. Figure 9 illustrates this scenario.

Before Hayabusa arrived at Itokawa, we though that it might be difficult to separate the effect of the solar radiation pressure from the gravitational attraction of Itokawa, because the gravity of Itokawa is very small. We also thought that it might be difficult to estimate the mass of Itokawa, because Hayabusa does not make revolution around Itokawa. However, these assumptions were not correct. We were able to determine the gravitational attraction from Itokawa fairly well.

What we were right is the fact that the orbit and attitude control by chemical thrusters would make it difficult to estimate the mass. Therefore, in future missions, we should be

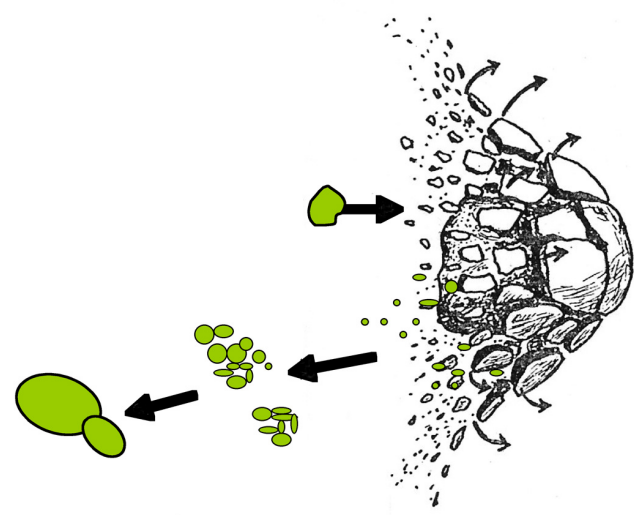

Figure 9. Possible origin of Itokawa. careful about this point. 


\section{Acknowledgments}

We think that Hayabusa mission was successful because many people joined forces to overcome many difficulties. Especially here we would like to express our appreciation to the navigators in Jet Propulsion Laboratory and the operators of Deep Space Network for their excellent assistance.

\section{References}

${ }^{1}$ Science, Vol.312, No.5778, 2 June 2006.

${ }^{2}$ Ikeda, H., Kominato, T., Masuoka, M., Ohnishi, T., and Yoshikawa, M., "Mass Estimation of Asteroid Itokawa by Using Range and Doppler Data Observed Near Gate Position," Second International Hayabusa Symposium, July 2006, p.8.

${ }^{3}$ Kominato, T., Matsuoka, M., Uo, M., Hashioto, T., and Kawaguchi, J., "Optical Hybrid Navigation in Hayabusa - Approach, Station Keeping \& Hovering," Advances in the Astronautical Sciences [CD-ROM], Vol. 124, AAS 06-210, AIAA/AAS, Tampa, Florida, 22-26 Jan. 2006.

${ }^{4}$ Abe, S., Mukai, T., Hirata, N., Barnouin-Jha, O. S., Cheng, A. F., Demura, H., Gaskell, R. W., Hashimoto, T., Hiraoka, K., Honda, T., Kubota, T., matsuoka, M., Mizuno, T., Nakamura, R., Scheeres, D. J., and Yoshikawa, M., "Mass and Local Topography Measurements of Itokawa by Hayabusa," Science, Vol. 312, No. 5778, 2006, pp.1344-1347.

${ }^{5}$ Scheeres, D. J., Gaskell, R., Abe, S., Barnouin-Jha, O., Hashimoto, T., Hirata, N., Ishiguro, M., Kawaguchi, J., Kominato, T., Kubota, T., Mukai, T., Saito, J., Shirakawa, K., Uo, M., and Yoshikawa, M., "The Actual Dynamical Environment about Itokawa," AIAA/AAS Astrodynamics Specialist Conference and Exhibit, Keystone, Colorado, August 2006, AIAA-2006-6661.

${ }^{6}$ Yano, H., Kubota, T., Miyamoto, H., Okada, T., Scheeres, D., Takagi, Y., Yoshida, K., Abe, M., Barnouin-Jha, O., Fujiwara, A., Hasegawa, S., Hasguniti, T., Ishiguro, M., Kato, M., Kawaguchi, J., Mukai, T., Saito, J., Sasaki, S., and Yoshikawa, M., "Touchdown of the Hayabusa Spacecraft at the Muses Sea on Itokawa," Science, Vol. 312, No. 5778, 2006, pp.1350-1353.

${ }^{7}$ Demura, H., Kobayashi, S., Nemoto, E., Matsumoto, N., Furuya, M., Yukishita, A., Muranaka, N., Morita, H., Shirakawa, K., Maruya, M., Ohyama, H., Uo, M., Kubota, T., Hashimoto, T., Kawaguchi, J., Fujiwara, A., Saito, J., Sasaki, S., Miyamoto, H., and Hirata, N., "Pole and Global Shape of 25143 Itokawa," Science, Vol. 312, No. 5778, 2006, pp.1347-1349.

${ }^{8}$ Fujiwara, A., Kawaguchi, J., Yeomans, D.K., Abe, M., Mukai, T., Okada, T., Saito, J., Yano, H., Yoshikawa, M., Scheeres, D. J., Barnouin-Jha, O., Cheng, A.F., Demura, H., Gaskell, R. W., Hirata, N., Ikeda, H., Kominato, T., Miyamoto, H., Nakamura, A. M., Nakamura, R., Sasaki, S., and Uesugi, K., "The Rubble-Pile Asteroid Itokawa as Observed by Hayabusa," Science, Vol. 312, No. 5778, 2006, pp.1330-1334. 